\section{Agregação dos fatores de risco cardiovascular: álcool, fumo, excesso de peso e sono de curta duração em adolescentes do estudo ERICA}

\author{
Aggregation of cardiovascular risk factors: alcohol, \\ smoking, excess weight, and short sleep duration \\ in adolescents in the ERICA study
}

Agregación de los factores de riesgo cardiovascular: alcohol, tabaco, exceso de peso y sueño de corta duración en adolescentes del estudio ERICA

\section{Resumo}

O objetivo foi analisar a agregação do consumo de álcool, tabaco, excesso de peso e sono curto em adolescentes brasileiros. Trata-se de estudo transversal, multicêntrico realizado com base em adolescentes participantes do Estudo de Riscos Cardiovasculares em Adolescentes (ERICA). A amostra foi composta por adolescentes que responderam completamente aos questionários sobre sono, tabaco e uso de bebidas alcoólicas, além de terem realizado antropometria com medidas de peso e estatura aferidas. A agregação foi analisada comparando a prevalência observada com a esperada dos fatores de risco em todas as possibilidades de agrupamento, e seus intervalos de 95\% de confiança. As análises foram realizadas no programa estatístico Stata 14, mediante uso do comando svy (survey) para dados de amostra complexa. São 73.624 adolescentes, 25,5\% dos estudantes apresentam excesso de peso e 24,2\% fazem uso de bebida alcoólica. A agregação dos quatro fatores de risco foi de $\mathrm{O} / \mathrm{E}=5,6 . \mathrm{A}$ prevalência de agregação dos três fatores foi maior naqueles com 15 a 17 anos $(P=4,8)$. Na análise de ORP (odds ratio de prevalência) da combinação de dois fatores de risco, observou-se que os fumantes têm 11,80 vezes mais chances de também beber quando comparados àqueles que não fumam e vice-versa, nas escolas privadas. Em relação à idade, adolescentes com 12 e 14 anos que fumam têm 15, 46 vezes mais chances de também beber e vice-versa. Adolescentes estudados apresentam a presença dos quatro fatores de forma agregada e há relação significativa entre o tabaco e o consumo de álcool.

Adolescente; Bebidas Alcoólicas; Tabaco; Sono; Fatores de Risco
Gabriela Oliveira 1

Thiago Luiz Nogueira da Silva 2

Isabel Batista da Silva 1

Evandro Silva Freire Coutinho 3

Katia Vergetti Bloch 2

Elizabete Regina Araujo de Oliveira 1

doi: 10.1590/0102-311X00223318

\section{Correspondência}

G. Oliveira

Universidade Federal do Espirito Santo.

Av. Marechal Campos 1468, Vitória, ES 29060-270, Brasil.

oli.gabriela@hotmail.com

1 Universidade Federal do Espírito Santo, Vitória, Brasil.

2 Instituto de Estudos em Saúde Coletiva, Universidade Federal do Rio de Janeiro, Rio de Janeiro, Brasil.

3 Escola Nacional de Saúde Pública Sergio Arouca, Fundação Oswaldo Cruz, Rio de Janeiro, Brasil. 


\section{Introdução}

As doenças cardiovasculares (DCV) são um problema de saúde relevante e global. Em 2012, ocorreram 17,5 milhões de mortes em todo o mundo, e se estima que 7,4 milhões morreram de doenças isquêmicas do coração e 6,7 milhões de acidente vascular cerebral (AVC). Mais de 80\% das mortes por DCV ocorrem em países de baixa e média renda 1 . Diversos são os fatores de risco apontados para DCV, e quanto mais fatores o indivíduo apresentar, isto é, quanto maior agregação ou acúmulo de fatores em um indivíduo, maior o seu risco de desenvolver uma DCV.

O uso excessivo de álcool, o tabagismo, o excesso de peso e o sono inadequado têm sido apontados como fatores de risco para as DCV 2,3. Um estudo realizado por Burke et al. 4 analisou a associação entre cigarro e DCV de 1971 a 2006, em uma população proveniente do Estudo Framingham. Os autores observaram que, ao longo de três décadas, homens que fumavam mantiveram um risco duas vezes maior de DCV em relação aos que não fumavam, enquanto nas mulheres o risco aumentou 1,6 vez. O uso de álcool consumido em grande quantidade tem sido apontado como fator de risco para pressão arterial elevada, obesidade e AVC, entre outras DCV. O excesso de peso, que inclui o sobrepeso e a obesidade, é visto como um problema de saúde pública crescente e disseminado. No Brasil, a pesquisa Vigilância de Fatores de Risco e Proteção para Doenças Crônicas por Inquérito Telefônico (Vigitel), de 2016, mostrou que o excesso de peso está presente em $53,8 \%$ da população e que a obesidade cresceu $60 \%$ em dez anos, passando de 11,8\% em 2006 para 18,9\% em 2016 5. Já o sono tem um papel restaurador, pois enquanto a pessoa dorme, o corpo trabalha para manter a função saudável do sistema cardiovascular, imunológico, digestivo e outros, além da saúde física 6 . O tempo de sono menor que o recomendado tem sido associado com maior uso de bebidas alcoólicas, tabaco e maconha 7, além de afetar o desempenho acadêmico e o número de abstenções na escola 8,9 . Nos últimos anos, têm sido realizados cada vez mais estudos abordando o sono, constatando seu importante papel na saúde humana e influência do atual estilo de vida a esse.

Tal cenário, com prevalências elevadas de fatores de risco cardiovascular em fases precoces da vida, no qual o estilo de vida do indivíduo durante sua adolescência afeta e influencia a sua saúde quando adulto, aponta para a necessidade de dimensionar os possíveis efeitos da exposição a mais de um fator na saúde cardiovascular de adolescentes. Estudos para conhecer a agregação de fatores de risco cardiovascular em adolescentes passaram a ser realizados e constataram a agregação dos diversos fatores de risco na vida dos adolescentes 10,11,12; vale ressaltar, no entanto, que estudos realizados com adolescente não analisaram a curta duração do sono agregado a outros fatores de risco.

Em razão da potencialização de fatores de riscos, quando agregados a outros, o interesse sobre como eles se encontram na população adolescente é grande em todo o mundo, visto que intervenções que tenham o foco em vários fatores podem favorecer a sua redução. Caso contrário, a possibilidade de os fatores se manterem na vida adulta é grande, o que agrava a ocorrência do indivíduo ter alguma DCV. A ocorrência desses fatores nesta fase da vida possivelmente surge devido ao estilo de vida que acaba sendo adotado, influenciado por seus pares, pelas tendências da moda e impulsividade por experiências novas.

O objetivo deste estudo foi descrever a prevalência de agregação de importantes fatores de risco cardiovascular (tabagismo, consumo de álcool, sono de curta duração e excesso de peso) em adolescentes brasileiros de 12 a 17 anos de idade.

\section{Métodos}

O presente artigo fez uso dos dados do Estudo de Riscos Cardiovasculares em Adolescentes (ERICA), realizado nos anos de 2013 e 2014. Trata-se de um estudo seccional, nacional, que investigou a prevalência de fatores de risco cardiovasculares e síndrome metabólica em uma amostra representativa de estudantes com idade entre 12 e 17 anos, de escolas públicas e privadas, de todas as capitais, do Distrito Federal e de cidades com mais de 100 mil habitantes do Brasil. A coleta dos dados foi realizada dentro da sala de aula com apoio de um coletor eletrônico de dados individual portátil, modelo LG GM750Q e supervisão da equipe do estudo. O questionário foi divido em blocos de acordo com temas de interesse da pesquisa (exemplo o "Bloco 6: uso de bebidas alcoólicas") e que possuíam alternativas para 
respostas. Detalhes sobre o delineamento amostral e o protocolo de coleta de dados do estudo foram descritos em publicações anteriores 13,14 .

A pesquisa foi aprovada pelo Comitê de Ética em Pesquisa (CEP) do Instituto de Estudos em Saúde Coletiva da Universidade Federal do Rio de Janeiro (IESC/UFRJ) e pelo CEP de cada Unidade da Federação. Os adolescentes participantes assinaram termo de assentimento e apresentaram o termo de consentimento livre e esclarecido assinado pelo responsável quando solicitado.

Dos 102.327 adolescentes elegíveis (com 12 a 17 anos de idade, sem deficiência física ou cognitiva, seja temporária ou permanente, e não grávidas), 73.624 preencheram completamente o questionário e realizaram antropometria 15 . Desses, 65.837 possuíam informações válidas sobre a duração do sono, além dos dados sobre tabagismo, álcool e excesso de peso.

\section{Variáveis de interesse}

Tabagismo e uso de álcool: foram considerados usuários de tabaco aqueles que fumaram pelo menos uma vez nos últimos 30 dias 16 e de bebidas alcoólicas aqueles que consumiram bebida alcoólica pelo menos uma vez nos últimos 30 dias 17 .

Sono: a duração do sono foi calculada pela média ponderada da hora de sono durante a semana (obtida pela diferença entre a hora de dormir e acordar durante a semana) e do final de semana (obtida pela diferença entre a hora de dormir e acordar no final de semana), mediante a seguinte fórmula: (duração do sono durante a semana x $5+$ duração do sono no final de semana $\times 2$ )/7. Os valores encontrados foram considerados válidos apenas se estivessem dentro dos limites $\geq 4$ horas e $\leq 14$ horas. Com isso, houve uma perda de 7.787 observações (10,6\%), seja pela inconsistência da duração do sono ou pela hora do sono informada (acordar e/ou dormir) incompatível com o horário escolar. A variável sono foi classificada como de curta duração para aqueles com $\geq 4$ horas até $<7$ horas de sono noturno 18 .

Excesso de peso: Essa variável teve como base a classificação do estado nutricional dos adolescentes com sobrepeso e obesidade, obtida pelo escore- $Z$ do índice de massa corporal (IMC) para idade segundo sexo ${ }^{19}$. O IMC foi obtido pela razão entre o peso $(\mathrm{kg})$ e o quadrado da estatura $(\mathrm{m})$, mediante antropométricas coletadas 14 . Foram classificados como excesso de peso os adolescentes com escore- $Z>1$. Covariáveis: a análise também inclui as variáveis sexo (feminino e masculino), idade (categorizada em faixas etárias de 12 a 14 anos e 15 a 17 anos), macrorregiões do Brasil (Norte, Nordeste, Centro-oeste, Sudeste e Sul) e rede de ensino (pública ou privada).

\section{Análise estatística}

Inicialmente, cada um dos quatro fatores de risco investigados, consumo de bebida alcoólica, tabaco, sono curto e excesso de peso, foi categorizado quanto à sua presença ou ausência, sendo calculadas suas prevalências e respectivos intervalos de 95\% de confiança (IC95\%). As prevalências foram estratificadas por sexo, idade (12 a 14 anos e 15 a 17 anos), tipo de escola (pública ou privada) e macrorregiões (Norte, Nordeste, Centro-oeste, Sudeste e Sul).

A variável agregação foi definida como o acúmulo, em um mesmo adolescente, de dois, três ou quatro fatores de risco cardiovascular de interesse: uso de álcool, tabaco, excesso de peso e sono curto. A princípio, foram estimadas as prevalências observada e esperada ao acaso das combinações possíveis de fatores de risco cardiovasculares. A prevalência esperada de cada combinação dos fatores foi calculada multiplicando-se as probabilidades individuais de cada fator. Razões entre valores observados e esperados ao acaso foram calculadas, assim como seus respectivos IC95\%. Quando o valor dessa razão excedia 1, considerou-se que havia agregação entre os fatores 20 . Posteriormente, foi calculado odds ratio de prevalência (ORP), usado para calcular a agregação de dois comportamentos, de acordo com a fórmula abaixo.

Número de entrevistados sem nenhum fator de risco $x$ Número de entrevistados com os fatores de risco $A$ e $B$

Número de entrevistados com um único fator de risco $x$ Número de entrevistados com o outro fator de risco

Um ORP de 2,5, por exemplo, indica que um indivíduo que apresenta um fator A tem uma chance 2,5 vezes maior de ter o fator $B$ do que o indivíduo que não tem o fator $A$.

As análises foram realizadas no programa estatístico Stata 14 (https://www.stata.com/), utilizando-se o comando svy (survey) para dados de amostra complexa. 


\section{Resultados}

A amostra foi composta por 73.624 estudantes, sendo $55,4 \%$ do sexo feminino. Cerca de $78,7 \%$ da amostra foi composta por adolescentes de escolas públicas, com concentração um pouco mais elevada na faixa etária de 14 a 17 anos. A Região Nordeste contribuiu com o maior número $(31,1 \%)$ de participantes, enquanto a Região Sul teve o menor número (12,7\%) (Tabela 1). O fator de risco do sono foi o único que apresentou perda de informação, correspondendo a 7.787 adolescentes $(10,6 \%)$ em relação ao total da amostra.

Na Tabela 2, encontrou-se que excesso de peso e consumo de álcool estavam presentes em cerca de $1 / 4$ da amostra total. Sono de curta duração foi referido por cerca de $18 \%$, enquanto o tabagismo apresentou menor prevalência (5\%). A prevalência de uso de bebida alcoólica e de tabaco foi maior nos adolescentes mais velhos, enquanto a prevalência de excesso de peso estava menor nos mais velhos, sendo mais prevalente nas escolas privadas, semelhante ao que ocorreu com sono de curta duração. A Região Sul apresentou a maior prevalência de adolescentes consumindo bebida alcoólica e com excesso de peso.

A agregação ou ocorrência conjunta dos quatro fatores de risco foi observada em apenas 0,3\% dos adolescentes, enquanto a prevalência de três fatores de risco se mostrou presente em 3,1\%. Esse padrão de agregação se diferenciou por idade e região, sendo maior na faixa etária de 15 a 17 anos e menor nas regiões Norte e Nordeste. A agregação para dois fatores, embora mais elevada do que a de três fatores, seguiu um padrão de distribuição padrão semelhante (Tabela 3).

As informações sobre a agregação (prevalência observada/esperada ao acaso) das diferentes combinações de fatores de risco cardiovascular se encontram na Tabela 4. Sendo a prevalência conjunta constatada 5,6 vezes maior do que a esperada ao acaso. Com relação à agregação de três fatores, a mais frequente foi tabaco, consumo de álcool e sono de curta duração $(\mathrm{O} / \mathrm{E}=5,6)$. A ocorrência de três fatores de risco para a qual não se verificou agregação foi tabaco, sono de curta duração e excesso de peso. No caso da agregação de dois fatores, o número observado superou o esperado ao acaso para tabaco e álcool $(\mathrm{O} / \mathrm{E}=2,5)$ assim como para álcool e sono de curta duração $(\mathrm{O} / \mathrm{E}=1,2)$.

A Tabela 5 traz o ORP para a associação de pares de fatores de risco. A associação de maior magnitude foi do tabaco e consumo de álcool (ORP $=11,80)$, sendo mais elevada em escolas privadas em comparação às públicas; aqueles que fumam têm 23,10 vezes mais chances de também beber quando

Tabela 1

Características sociodemográficas e fatores de risco cardiovascular dos adolescentes participantes do Estudo de Riscos Cardiovasculares em Adolescentes (ERICA). Brasil, 2013-2014.

\begin{tabular}{|c|c|c|c|c|c|c|}
\hline \multirow[t]{2}{*}{ Características } & \multicolumn{2}{|c|}{ Feminino } & \multicolumn{2}{|c|}{ Masculino } & \multicolumn{2}{|c|}{ Total } \\
\hline & $\mathbf{n}$ & $\%$ & $\mathbf{n}$ & $\%$ & $\mathrm{n}$ & $\%$ \\
\hline Sexo & 40.803 & 55,42 & 32.821 & 44,58 & 73.624 & 100,00 \\
\hline \multicolumn{7}{|l|}{ Faixa etária (anos) } \\
\hline $12-14$ & 18.554 & 45,47 & 15.224 & 46,38 & 33.778 & 45,88 \\
\hline $15-17$ & 22.249 & 54,53 & 17.597 & 53,62 & 39.846 & 54,12 \\
\hline \multicolumn{7}{|l|}{ Escola } \\
\hline Pública & 32.294 & 79,15 & 25.676 & 78,23 & 57,97 & 78,74 \\
\hline Privada & 8.509 & 20,85 & 7.145 & 21,77 & 15,654 & 21,26 \\
\hline \multicolumn{7}{|l|}{ Região } \\
\hline Norte & 8.174 & 20,03 & 6.822 & 20,79 & 14.996 & 20,37 \\
\hline Nordeste & 12.737 & 31,22 & 10.127 & 30,86 & 22.864 & 31,06 \\
\hline Centro-oeste & 5.573 & 13,66 & 4.031 & 12,28 & 9.604 & 13,04 \\
\hline Sudeste & 9.360 & 22,94 & 7.481 & 22,79 & 16.841 & 22,87 \\
\hline Sul & 4.959 & 12,15 & 4.360 & 13,28 & 9.319 & 12,66 \\
\hline
\end{tabular}




\section{Tabela 2}

Prevalências (\%) * dos fatores de risco tabaco, consumo de álcool, sono de curta duração e excesso de peso segundo sexo, faixa etária, tipo de escola e macrorregião. Brasil, 2013-2014.

\begin{tabular}{|c|c|c|c|c|c|c|c|c|}
\hline \multirow[t]{2}{*}{ Características } & \multicolumn{2}{|c|}{ Tabagismo } & \multicolumn{2}{|c|}{$\begin{array}{c}\text { Consumo de } \\
\text { álcool }\end{array}$} & \multicolumn{2}{|c|}{$\begin{array}{l}\text { Sono de curta } \\
\text { duração }\end{array}$} & \multicolumn{2}{|c|}{ Excesso de peso } \\
\hline & $\%$ & IC95\% & $\%$ & IC95\% & $\%$ & IC95\% & $\%$ & IC95\% \\
\hline Todos & 5,2 & $4,8-5,6$ & 24,2 & $23,2-25,2$ & 17,8 & $16,9-18,7$ & 25,5 & $24,4-26,6$ \\
\hline \multicolumn{9}{|l|}{ Sexo } \\
\hline Feminino & 4,8 & $4,4-5,3$ & 24,5 & $23,4-25,7$ & 17,3 & $16,3-18,4$ & 25,3 & $23,9-26,8$ \\
\hline Masculino & 5,5 & $4,9-6,2$ & 23,8 & $22,1-25,7$ & 18,2 & $17,1-19,4$ & 25,7 & $24,4-27,1$ \\
\hline \multicolumn{9}{|l|}{ Faixa etária (anos) } \\
\hline $12-14$ & 3,3 & $2,8-3,8$ & 16,4 & $15,2-17,5$ & 11,1 & $10,2-12,0$ & 28,1 & $26,3-30,0$ \\
\hline $15-17$ & 7,3 & $6,6-8,0$ & 33,0 & $31,3-34,7$ & 25,3 & $23,9-26,7$ & 22,6 & $21,3-23,9$ \\
\hline \multicolumn{9}{|l|}{ Escola } \\
\hline Pública & 5,4 & $5-5,9$ & 24,4 & $23,3-25,5$ & 17,5 & $16,4-18,6$ & 24,1 & $22,9-25,3$ \\
\hline Privada & 4,1 & $3-5,7$ & 23,4 & $19,8-27,4$ & 19,1 & $15,9-22,8$ & 31,7 & $29,6-33,8$ \\
\hline \multicolumn{9}{|l|}{ Região } \\
\hline Norte & 5,2 & $4,5-6,0$ & 17,9 & $16,9-19,0$ & 16,9 & $15,4-18,6$ & 21,8 & $20,6-23,1$ \\
\hline Nordeste & 4,4 & $3,6-5,3$ & 18,9 & $17,3-20,7$ & 14,9 & $13,5-16,5$ & 23,6 & $21,7-25,7$ \\
\hline Centro-oeste & 5,7 & $4,7-6,9$ & 26,5 & $24,6-28,5$ & 17,1 & $15,6-18,7$ & 23,5 & $22,2-24,9$ \\
\hline Sudeste & 5,1 & $4,5-5,8$ & 25,7 & $23,9-27,6$ & 19,6 & $18,1-21,1$ & 26,1 & $24,2-28,1$ \\
\hline Sul & 6,5 & $5,4-7,7$ & 30,3 & $28,4-32,3$ & 16,4 & $14,1-19,1$ & 30,2 & $28,3-32,1$ \\
\hline
\end{tabular}

IC95\%: intervalo de 95\% de confiança.

* Ajustadas segundo o delineamento amostral do estudo.

comparados com aqueles que não fumam e vice-versa, enquanto nas escolas públicas esse número cai para 10,69, e na faixa etária de 12 a 14 anos $(\mathrm{ORP}=15,46)$ e faixa etária de 12 a 14 anos em comparação com a faixa de 15 a 17 anos. A agregação entre tabaco e sono ocupou o segundo lugar, com uma magnitude bem inferior ( $\mathrm{ORP}=2,11$ ), sem variações importantes por subgrupo. Em terceiro lugar está a associação entre o álcool e o sono curto $(\mathrm{ORP}=1,95)$. As associações de tabagismo e excesso de peso, consumo de álcool e excesso de peso, sono de curta duração e excesso de peso não apresentaram estimativas de ORP estatisticamente significantes ( $O R P=1,04 ; 0,99 ; 1,09$, respectivamente), uma vez que seus IC95\% incluíram o valor nulo $(\mathrm{ORP}=1,00)$ (Tabela 5).

\section{Discussão}

Entre os quatro fatores de risco cardiovascular investigados, o excesso de peso foi o que apresentou maior prevalência, presente em cerca de 1/4 da população de adolescentes brasileiros, particularmente nos estudantes mais jovens (10 a 14 anos). Esse resultado é consistente com os achados da Pesquisa de Orçamentos Familiares (POF), realizada entre 2008 e 2009, em que 1/5 dos adolescentes brasileiros (de ambos os sexos) foi diagnosticado com excesso de peso, com tendência de essa presença ser mais frequente no meio urbano do que no meio rural, além de sua prevalência aumentar de acordo com a renda 21 . A alta presença de tal fator caracteriza uma transição nutricional que vem ocorrendo no país, com o alto consumo de alimentos calóricos e de baixa qualidade nutricional. O excesso de peso está ligado de forma direta ao desencadeamento de DCV, diabetes e até mesmo neoplasias 22,23,24. Zheng et al. 23 constataram risco significativo de desenvolvimento de DCV, além de outras morbidades, analisando o ganho de peso de indivíduos no início da vida adulta até aos 55 anos, por meio de duas coortes. 


\section{Tabela 3}

Prevalência (\%) * de agregação dos fatores de risco: tabaco, consumo de álcool, sono de curta duração e excesso de peso. Brasil, 2013-2014.

\begin{tabular}{|c|c|c|c|c|c|c|c|c|}
\hline \multirow[t]{2}{*}{ Características } & \multicolumn{2}{|c|}{1 fator de risco } & \multicolumn{2}{|c|}{2 fatores de risco } & \multicolumn{2}{|c|}{3 fatores de risco } & \multicolumn{2}{|c|}{4 fatores de risco } \\
\hline & $\%$ & IC95\% & $\%$ & IC95\% & $\%$ & IC95\% & $\%$ & IC95\% \\
\hline Todos & 36,4 & $35,5-37,3$ & 12,8 & $12,1-13,5$ & 3,1 & $2,7-3,5$ & 0,3 & $0,2-0,5$ \\
\hline \multicolumn{9}{|l|}{ Sexo } \\
\hline Feminino & 35,6 & $34,4-36,8$ & 13,1 & $12,3-14,0$ & 2,9 & $2,5-3,4$ & 0,3 & $0,2-0,6$ \\
\hline Masculino & 37,2 & $36,0-38,4$ & 12,5 & $11,5-13,5$ & 3,3 & $2,8-3,9$ & 0,3 & $0,2-0,5$ \\
\hline \multicolumn{9}{|l|}{ Faixa etária (anos) } \\
\hline $12-14$ & 36,0 & $34,6-37,5$ & 8,6 & $7,9-9,5$ & 1,6 & $1,3-1,9$ & 0,2 & $0,1-0,5$ \\
\hline $15-17$ & 36,8 & $35,4-38,1$ & 17,5 & $16,4-18,6$ & 4,8 & $4,2-5,5$ & 0,5 & $0,3-0,7$ \\
\hline \multicolumn{9}{|l|}{ Escola } \\
\hline Pública & 35,8 & $34,9-36,9$ & 12,7 & $11,9-13,4$ & 3,0 & $2,5-3,4$ & 0,3 & $0,2-0,5$ \\
\hline Privada & 38,8 & $36,9-40,6$ & 13,5 & $10,8-16,8$ & 3,8 & $2,9-5,1$ & 0,2 & $0,1-0,4$ \\
\hline \multicolumn{9}{|l|}{ Região } \\
\hline Norte & 34,4 & $33,3-35,6$ & 10,4 & $9,7-11,2$ & 2,0 & $1,7-2,3$ & 0,2 & $0,1-0,3$ \\
\hline Nordeste & 35,0 & $33,4-36,8$ & 10,2 & $9,1-11,4$ & 1,9 & $1,5-2,4$ & 0,2 & $0,1-0,4$ \\
\hline Centro-oeste & 35,3 & $33,8-36,8$ & 12,7 & $11,6-14,0$ & 3,7 & $2,9-4,8$ & 0,2 & $0,1-0,4$ \\
\hline Sudeste & 36,8 & $35,3-38,3$ & 13,7 & $12,5-15,1$ & 3,6 & $3,0-4,4$ & 0,3 & $0,2-0,5$ \\
\hline Sul & 39,1 & $36,9-41,4$ & 15,5 & $14,3-16,8$ & 3,6 & $3,0-4,5$ & 0,6 & $0,2-2,1$ \\
\hline
\end{tabular}

IC95\%: intervalo de $95 \%$ de confiança.

* Ajustadas segundo o delineamento amostral do estudo.

Tabela 4

Agregação dos quatro fatores de risco em adolescentes brasileiros de 12 a 17 anos. Brasil, 2013-2014.

\begin{tabular}{|c|c|c|c|c|c|c|c|c|}
\hline Fatores & $\mathbf{T}$ & A & SCD & EP & $\mathrm{O}(\%)$ & $E(\%)$ & O/E & IC95\% \\
\hline 4 & + & + & + & + & 0,3 & 0,1 & 5,6 & $4,8-6,4$ \\
\hline \multirow[t]{4}{*}{3} & + & + & + & - & 0,9 & 0,2 & 5,6 & $5,1-6,0$ \\
\hline & + & + & - & + & 0,8 & 0,3 & 2,9 & $2,7-3,2$ \\
\hline & + & - & + & + & 0,1 & 0,2 & 0,5 & $0,4-0,7$ \\
\hline & - & + & + & + & 1,3 & 1,0 & 1,3 & $1,2-1,4$ \\
\hline \multirow[t]{6}{*}{2} & + & + & - & - & 1,9 & 0,8 & 2,5 & $2,4-2,7$ \\
\hline & + & - & + & - & 0,2 & 0,5 & 0,4 & $0,4-0,5$ \\
\hline & + & - & - & + & 0,2 & 0,8 & 0,2 & $0,2-0,3$ \\
\hline & - & + & + & - & 3,7 & 3,0 & 1,2 & $1,2-1,3$ \\
\hline & - & + & - & + & 3,7 & 4,8 & 0,8 & $0,7-0,8$ \\
\hline & - & - & + & + & 3,0 & 3,3 & 0,9 & $0,9-1.0$ \\
\hline \multirow[t]{4}{*}{1} & + & - & - & - & 0,7 & 2,4 & 0,3 & $0,3-0,3$ \\
\hline & - & + & - & - & 11,5 & 14,1 & 0,8 & $0,8-0,8$ \\
\hline & - & - & + & - & 8,1 & 9,5 & 0,9 & $0,8-0,9$ \\
\hline & - & - & - & + & 16,0 & 15,1 & 1,1 & $1,0-1,1$ \\
\hline 0 & - & - & - & - & 47,4 & 44,0 & 1,1 & $1,1-1,1$ \\
\hline
\end{tabular}

A: consumo de álcool; E: esperado; EP: excesso de peso; IC95\%: intervalo de 95\% de confiança; O: observado; O/E: razão observado/esperado; SCD: sono de curta duração; T: tabagismo. 
Tabela 5

Odds ratio de prevalência (ORP) de dois fatores de risco cardiovascular nos adolescentes. Brasil, 2013-2014.

\begin{tabular}{|c|c|c|c|c|c|c|}
\hline \multirow[t]{2}{*}{ Características } & \multicolumn{2}{|c|}{ Tabagismo e consumo de álcool } & \multicolumn{2}{|c|}{ Tabagismo e sono de curta duração } & \multicolumn{2}{|c|}{ Tabagismo e excesso de peso } \\
\hline & $\begin{array}{c}\% \\
\text { (IC95\%) }\end{array}$ & $\begin{array}{c}\text { ORP } \\
\text { (IC95\%) }\end{array}$ & $\begin{array}{c}\% \\
\text { (IC95\%) }\end{array}$ & $\begin{array}{c}\text { ORP } \\
\text { (IC95\%) }\end{array}$ & $\begin{array}{c}\% \\
\text { (IC95\%) }\end{array}$ & $\begin{array}{c}\text { ORP } \\
\text { (IC95\%) }\end{array}$ \\
\hline Todos & $\begin{array}{c}3,9 \\
(3,6-4,3)\end{array}$ & $\begin{array}{c}11,80 \\
(10,01-13,90)\end{array}$ & $\begin{array}{c}1,6 \\
(1,4-1,8)\end{array}$ & $\begin{array}{c}2,11 \\
(1,76-2,52)\end{array}$ & $\begin{array}{c}1,4 \\
(1,2-1,6)\end{array}$ & $\begin{array}{c}1,04 \\
(0,86-1,27)\end{array}$ \\
\hline \multicolumn{7}{|l|}{ Sexo } \\
\hline Feminino & $\begin{array}{c}3,7 \\
(3,3-4,2)\end{array}$ & $\begin{array}{c}12,28 \\
(9,62-15,68)\end{array}$ & $\begin{array}{c}1,4 \\
(1,2-1,7)\end{array}$ & $\begin{array}{c}2,10 \\
(1,66-2,67)\end{array}$ & $\begin{array}{c}1,4 \\
(1,1-1,7)\end{array}$ & $\begin{array}{c}1,20 \\
(0,91-1,59)\end{array}$ \\
\hline Masculino & $\begin{array}{c}4,2 \\
(3,6-4,8)\end{array}$ & $\begin{array}{c}11,45 \\
(9,09-14,43)\end{array}$ & $\begin{array}{c}1,7 \\
(1,4-2,1)\end{array}$ & $\begin{array}{c}2,10 \\
(1,61-2,75)\end{array}$ & $\begin{array}{c}1,3 \\
(1,0-1,7)\end{array}$ & $\begin{array}{c}0,91 \\
(0,70-1,17)\end{array}$ \\
\hline \multicolumn{7}{|l|}{ Faixa etária (anos) } \\
\hline $12-14$ & $\begin{array}{c}2,4 \\
(2,0-2,9)\end{array}$ & $\begin{array}{c}15,46 \\
(11,59-20,62)\end{array}$ & $\begin{array}{c}0,7 \\
(0,5-1,0)\end{array}$ & $\begin{array}{c}2,34 \\
(1,64-3,35)\end{array}$ & $\begin{array}{c}0,9 \\
(0,6-1,2)\end{array}$ & $\begin{array}{c}0,91 \\
(0,65-1,27)\end{array}$ \\
\hline $15-17$ & $\begin{array}{c}5,7 \\
(5,1-6,4)\end{array}$ & $\begin{array}{c}8,59 \\
(7,14-10,34)\end{array}$ & $\begin{array}{c}2,5 \\
(2,1-2,9)\end{array}$ & $\begin{array}{c}1,62 \\
(1,31-1,99)\end{array}$ & $\begin{array}{c}1,9 \\
(1,6-2,3)\end{array}$ & $\begin{array}{c}1,24 \\
(0,98-1,57)\end{array}$ \\
\hline \multicolumn{7}{|l|}{ Escola } \\
\hline Pública & $\begin{array}{c}4,0 \\
(3,6-4,5)\end{array}$ & $\begin{array}{c}10,69 \\
(8,94-12,78)\end{array}$ & $\begin{array}{c}1,6 \\
(1,4-1,9)\end{array}$ & $\begin{array}{c}2,13 \\
(1,75-2,59)\end{array}$ & $\begin{array}{c}1,4 \\
(1,2-1,6)\end{array}$ & $\begin{array}{c}1,09 \\
(0,88-1,35)\end{array}$ \\
\hline Privada & $\begin{array}{c}3,6 \\
(2,6-4,9)\end{array}$ & $\begin{array}{c}23,10 \\
(16,10-33,16)\end{array}$ & $\begin{array}{c}1,3 \\
(0,9-1,9)\end{array}$ & $\begin{array}{c}2,04 \\
(1,27-3,27)\end{array}$ & $\begin{array}{c}1,3 \\
(0,8-1,9)\end{array}$ & $\begin{array}{c}0,93 \\
(0,60-1,46)\end{array}$ \\
\hline \multicolumn{7}{|l|}{ Região } \\
\hline Norte & $\begin{array}{c}3,7 \\
(3,1-4,3)\end{array}$ & $\begin{array}{c}13,36 \\
(10,91-16,36)\end{array}$ & $\begin{array}{c}1,1 \\
(0,9-1,4)\end{array}$ & $\begin{array}{c}1,39 \\
(1,09-1,77)\end{array}$ & $\begin{array}{c}1,0 \\
(0,8-1,4)\end{array}$ & $\begin{array}{c}0,87 \\
(0,66-1,16)\end{array}$ \\
\hline Nordeste & $\begin{array}{c}3,0 \\
(2,5-3,7)\end{array}$ & $\begin{array}{c}11,07 \\
(7,95-15,42)\end{array}$ & $\begin{array}{c}1,1 \\
(0,8-1,4)\end{array}$ & $\begin{array}{c}1,94 \\
(1,39-2,69)\end{array}$ & $\begin{array}{c}1,0 \\
(0,7-1,3)\end{array}$ & $\begin{array}{c}0,92 \\
(0,70-1,22)\end{array}$ \\
\hline Centro-oeste & $\begin{array}{c}4,5 \\
(3,6-5,7)\end{array}$ & $\begin{array}{c}12,86 \\
(9,08-18,21)\end{array}$ & $\begin{array}{c}1,5 \\
(1,0-2,1)\end{array}$ & $\begin{array}{c}1,72 \\
(1,16-2,55)\end{array}$ & $\begin{array}{c}1,7 \\
(1,3-2,3)\end{array}$ & $\begin{array}{c}1,40 \\
(1,09-1,81)\end{array}$ \\
\hline Sudeste & $\begin{array}{c}3,9 \\
(3,3-4,6)\end{array}$ & $\begin{array}{c}11,30 \\
(8,70-14,67)\end{array}$ & $\begin{array}{c}1,7 \\
(1,4-2,1)\end{array}$ & $\begin{array}{c}2,20 \\
(1,64-2,95)\end{array}$ & $\begin{array}{c}1,4 \\
(1,1-1,8)\end{array}$ & $\begin{array}{c}1,09 \\
(0,78-1,51)\end{array}$ \\
\hline Sul & $\begin{array}{c}5,4 \\
(4,4-6,7)\end{array}$ & $\begin{array}{c}14,21 \\
(8,32-24,26)\end{array}$ & $\begin{array}{c}2,2 \\
(1,4-3,2)\end{array}$ & $\begin{array}{c}2,76 \\
(1,83-4,16)\end{array}$ & $\begin{array}{c}1,8 \\
(1,3-2,7)\end{array}$ & $\begin{array}{c}0,91 \\
(0,54-1,54)\end{array}$ \\
\hline
\end{tabular}

(continua)

O consumo de bebida alcoólica vem em seguida e apresenta uma elevada prevalência (24,2\%). A Pesquisa Nacional de Saúde do Escolar (PeNSE) de 201525 demonstrou que 54,3\% dos adolescentes com faixa etária de 13 a 15 anos já haviam experimentado uma dose de bebida alcoólica e $73 \%$ dos escolares com 16 e 17 anos, também já ingeriram bebida alcoólica. Quanto à ocorrência de embriaguez, 21,4\% dos escolares já haviam sofrido algum episódio de embriaguez na vida. Vale ressaltar o alerta que esse dado nos apresenta, pois o consumo de bebidas alcóolicas no Brasil só é legalmente permitido após os 18 anos e, mesmo com as políticas públicas, tanto a venda quanto a compra por essa população continua ocorrendo.

Quando o indivíduo realiza o consumo de álcool junto com o fumo, ocorre uma interação sinérgica entre eles e aumenta o risco do desenvolvimento de doenças (isso em patologias em que o fumo também é um fator de risco), em comparação àqueles que só bebem ou só fumam 26. Esse dado é importante quando se leva em conta que as agregações de maior magnitude envolveram nesta amostra tabaco e consumo de álcool. Essa associação tem sido reportada por outros autores em estudos com adolescentes 27,28,29 e adultos 20,30, fato que demonstra que a agregação de tais fatores influenciará no desenvolvimento de outras doenças crônicas não transmissíveis além das DCV. 
Tabela 5 (continuação)

\begin{tabular}{|c|c|c|c|c|c|c|}
\hline \multirow[t]{2}{*}{ Características } & \multicolumn{2}{|c|}{$\begin{array}{l}\text { Consumo de álcool e sono de curta } \\
\text { duração }\end{array}$} & \multicolumn{2}{|c|}{$\begin{array}{l}\text { Consumo de álcool e excesso de } \\
\text { peso }\end{array}$} & \multicolumn{2}{|c|}{$\begin{array}{c}\text { Sono de curta duração e excesso } \\
\text { de peso }\end{array}$} \\
\hline & $\begin{array}{c}\% \\
(\text { IC95\%) }\end{array}$ & $\begin{array}{c}\text { ORP } \\
\text { (IC95\%) }\end{array}$ & $\begin{array}{c}\% \\
\text { (IC95\%) }\end{array}$ & $\begin{array}{l}\text { ORP } \\
\text { (IC95\%) }\end{array}$ & $\begin{array}{c}\% \\
\text { (IC95\%) }\end{array}$ & $\begin{array}{c}\text { ORP } \\
\text { (IC95\%) }\end{array}$ \\
\hline Todos & $\begin{array}{c}6,3 \\
(5,7-6,8)\end{array}$ & $\begin{array}{c}1,95 \\
(1,73-2,20)\end{array}$ & $\begin{array}{c}6,1 \\
(5,7-6,6)\end{array}$ & $\begin{array}{c}0,99 \\
(0,91-1,08)\end{array}$ & $\begin{array}{c}4,8 \\
(4,4-5,2)\end{array}$ & $\begin{array}{c}1,09 \\
(0,99-1,20)\end{array}$ \\
\hline \multicolumn{7}{|l|}{ Sexo } \\
\hline Feminino & $\begin{array}{c}6,2 \\
(5,5-7,0)\end{array}$ & $\begin{array}{c}1,96 \\
(1,70-2,26)\end{array}$ & $\begin{array}{c}6,4 \\
(5,8-7,1)\end{array}$ & $\begin{array}{c}1,06 \\
(0,94-1,21)\end{array}$ & $\begin{array}{c}4,7 \\
(4,2-5,2)\end{array}$ & $\begin{array}{c}1,12 \\
(0,98-1,29)\end{array}$ \\
\hline Masculino & $\begin{array}{c}6,3 \\
(5,7-7,0)\end{array}$ & $\begin{array}{c}1,95 \\
(1,63-2,34)\end{array}$ & $\begin{array}{c}5,9 \\
(5,2-6,5)\end{array}$ & $\begin{array}{c}0,92 \\
(0,80-1,07)\end{array}$ & $\begin{array}{c}4,9 \\
(4,3-5,5)\end{array}$ & $\begin{array}{c}1,07 \\
(0,90-1,26)\end{array}$ \\
\hline \multicolumn{7}{|l|}{ Faixa etária (anos) } \\
\hline $12-14$ & $\begin{array}{c}2,7 \\
(2,3-3,1)\end{array}$ & $\begin{array}{c}1,73 \\
(1,42-2,10)\end{array}$ & $\begin{array}{c}4,3 \\
(3,9-4,9)\end{array}$ & $\begin{array}{c}0,91 \\
(0,78-1,07)\end{array}$ & $\begin{array}{c}3,5 \\
(3,1-4,1)\end{array}$ & $\begin{array}{c}1,23 \\
(1,06-1,43)\end{array}$ \\
\hline $15-17$ & $\begin{array}{c}10,3 \\
(9,4-11,4)\end{array}$ & $\begin{array}{c}1,58 \\
(1,39-1,80)\end{array}$ & $\begin{array}{c}8,2 \\
(7,4-9,1)\end{array}$ & $\begin{array}{c}1,20 \\
(1,06-1,37)\end{array}$ & $\begin{array}{c}6,2 \\
(5,6-6,8)\end{array}$ & $\begin{array}{c}1,15 \\
(1,02-1,30)\end{array}$ \\
\hline \multicolumn{7}{|l|}{ Escola } \\
\hline Pública & $\begin{array}{c}6,0 \\
(5,4-6,7)\end{array}$ & $\begin{array}{c}1,84 \\
(1,59-2,12)\end{array}$ & $\begin{array}{c}6,1 \\
(5,6-6,6)\end{array}$ & $\begin{array}{c}1,06 \\
(0,97-1,16)\end{array}$ & $\begin{array}{c}4,4 \\
(3,9-4,9)\end{array}$ & $\begin{array}{c}1,07 \\
(0,96-1,18)\end{array}$ \\
\hline Privada & $\begin{array}{c}7,3 \\
(5,8-9,2)\end{array}$ & $\begin{array}{c}2,53 \\
(2,09-3,07)\end{array}$ & $\begin{array}{c}6,4 \\
(5,2-8,0)\end{array}$ & $\begin{array}{c}0,77 \\
(0,63-0,96)\end{array}$ & $\begin{array}{c}6,6 \\
(5,4-8,0)\end{array}$ & $\begin{array}{c}1,16 \\
(0,93-1,46)\end{array}$ \\
\hline \multicolumn{7}{|l|}{ Região } \\
\hline Norte & $\begin{array}{c}3,9 \\
(3,4-4,4)\end{array}$ & $\begin{array}{c}1,46 \\
(1,27-1,69)\end{array}$ & $\begin{array}{c}3,4 \\
(3-3,9)\end{array}$ & $\begin{array}{c}0,83 \\
(0,71-0,97)\end{array}$ & $\begin{array}{c}4,2 \\
(3,7-4,8)\end{array}$ & $\begin{array}{c}1,24 \\
(1,09-1,41)\end{array}$ \\
\hline Nordeste & $\begin{array}{c}4,1 \\
(3,4-4,9)\end{array}$ & $\begin{array}{c}1,78 \\
(1,49-2,13)\end{array}$ & $\begin{array}{c}4,6 \\
(4,0-5,2)\end{array}$ & $\begin{array}{c}1,03 \\
(0,83-1,28)\end{array}$ & $\begin{array}{c}3,3 \\
(2,8-3,9)\end{array}$ & $\begin{array}{c}0,91 \\
(0,78-1,05)\end{array}$ \\
\hline Centro-oeste & $\begin{array}{c}6,7 \\
(5,8-7,9)\end{array}$ & $\begin{array}{c}2,08 \\
(1,72-2,53)\end{array}$ & $\begin{array}{c}6,2 \\
(5,3-7,2)\end{array}$ & $\begin{array}{c}0,99 \\
(0,83-1,17)\end{array}$ & $\begin{array}{c}4,6 \\
(3,8-5,6)\end{array}$ & $\begin{array}{c}1,25 \\
(1,01-1,55)\end{array}$ \\
\hline Sudeste & $\begin{array}{c}7,3 \\
(6,4-8,4)\end{array}$ & $\begin{array}{c}2,03 \\
(1,65-2,49)\end{array}$ & $\begin{array}{c}6,8 \\
(6,0-7,7)\end{array}$ & $\begin{array}{c}1,02 \\
(0,89-1,16)\end{array}$ & $\begin{array}{c}5,5 \\
(4,8-6,2)\end{array}$ & $\begin{array}{c}1,12 \\
(0,96-1,31)\end{array}$ \\
\hline Sul & $\begin{array}{c}7,1 \\
(6,0-8,4)\end{array}$ & $\begin{array}{c}1,99 \\
(1,66-2,38)\end{array}$ & $\begin{array}{c}8,3 \\
(7,4-9,3)\end{array}$ & $\begin{array}{c}0,82 \\
(0,69-0,97)\end{array}$ & $\begin{array}{c}5,1 \\
(4,1-6,5)\end{array}$ & $\begin{array}{c}1,07 \\
(0,85-1,34)\end{array}$ \\
\hline
\end{tabular}

IC95\%: intervalo de 95\% de confiança.

Cureau et al. 27 analisaram a agregação de fatores de risco para doenças não transmissíveis em uma população de adolescentes de Santa Maria (Rio Grande do Sul) e verificaram que tanto o tabaco quanto o álcool estavam presentes na agregação juntamente com outros fatores, em ambos os sexos. A agregação do tabaco e álcool com outros fatores de risco também foi verificada em estudantes do Ensino Médio da rede pública estadual de Caruaru (Pernambuco) 28, assim como no estudo realizado por Oliveira et al. ${ }^{29}$, com 1.039 adolescentes com idade entre 13 e 19 anos, estudantes do Ensino Médio de escolas públicas e privadas do Rio de Janeiro.

Análises de agregação de fatores de risco que incluem o sono de curta duração em adolescentes são menos frequentes, o que é um diferencial neste estudo. Seo et al. 31, em 2018, investigaram a agregação de fatores de risco em crianças e adolescentes em um estudo de coorte, e observaram a presença de agregação do sono de curta duração com outros fatores de risco (excesso de peso/obesidade, baixo nível socioeconômico e histórico familiar para DCV) para DCV.

O presente estudo não atestou uma diferença importante na agregação dos fatores de risco por sexo (Tabela 3). Entretanto, houve maior agregação de fatores de risco em adolescentes mais velhos, o que também foi relatado por outros estudos 32,33,34. No estudo realizado por Brito et al. 32 em 2015, com 4.207 estudantes de Pernambuco, verifica-se que os adolescentes mais velhos apresentavam chance $17 \%$ maior de agregação de fatores de risco, quando comparado aos mais novos. Isso pode ocorrer por conta do fato de maior independência que esses indivíduos conquistam com o passar da idade. 
Os resultados sobre agregação de fatores de risco mostrados neste estudo assemelham-se ao resultado de outras pesquisas que também abordam fatores de risco de doenças crônicas 10,34 e até mesmo aqueles que abordam fatores não necessariamente ligados a risco cardiovascular, na população adolescente 28,35. Em Pernambuco, 58,5\% dos adolescentes com idades de 14 a 19 anos estavam expostos simultaneamente a dois ou mais fatores de risco 32. Em Caxias do Sul (Rio Grande do Sul), a prevalência dos fatores de risco para doenças crônicas não transmissíveis também foi alta (60\%) 33 .

A análise de ORP, que estima o quanto a presença de um fator de risco aumenta a chance da ocorrência de outro comportamento de risco, mostrou valores de maior magnitude para a combinação de consumo de álcool e tabaco. Essa combinação teve 15,45 vezes mais chances de ocorrer nos adolescentes de 12 e 14 anos; situação preocupante que expõe vulnerabilidade social com acesso precoce a drogas que são legalmente restritas a maiores de 18 anos. Também se verificou uma relação significativa entre o tabaco e o consumo de álcool para estudantes de escolas privadas. Uma explicação possível seria o maior poder econômico e o mais fácil acesso. A agregação em adolescentes de Pernambuco foi mais alta entre aqueles que apresentavam maior renda familiar 28. Porém, esse achado não é consistente. Silva et al. 33 observaram essa relação naqueles com menor renda.

Wu et al. 36 constataram que os adolescentes que apresentavam maior consumo de álcool e tabaco tinham taxas mais elevadas de relações sexuais precoce, sem uso de preservativo e em forma de comércio certificado pelo recebimento de dinheiro. Atitudes preocupantes que podem gerar outros problemas de saúde pública como a aumento de transmissão de doenças sexualmente transmissíveis (incluindo aids) e gravidez na adolescência.

Um dos pontos fortes deste estudo é a investigação da agregação dos fatores de risco na população adolescente porque grande parte dos estudos que aborda tal população se limita a analisar os fatores isoladamente.

Limitações do estudo podem ser levantadas como a análise com base em medidas autorrelatadas, que podem ser subestimadas pelos adolescentes por causa da não aceitação social, isso no que tange ao consumo de álcool e tabagismo. Já a análise do tipo de agregação tem como limitação a necessidade de resposta de todos os fatores investigados, a perda de 10,6\% dos dados ocorreu devido à dificuldade de os adolescentes responderem adequadamente sobre a duração do sono (hora de acordar e dormir). Nos últimos anos, diversos estudos 11,12,28 abordaram a agregação de diferentes fatores de risco de estilo de vida, contudo é difícil comparar esses estudos à medida que se utilizam diferentes combinações de fatores de risco, pontos de corte e população. Mas conhecer como esses fatores de risco para a saúde humana se apresentam, comportam e interagem é importante para a elaboração de estratégias de prevenção de doenças crônicas que são relevantes para a saúde pública. O envelhecimento populacional tornam as doenças crônicas de elevada prevalência um impacto econômico para os países.

Compreender a real situação da população adolescente brasileira quanto à agregação de fatores de risco é um passo essencial visto que as DCV são multicausais, além de contribuir para fomentar ações educativas e de saúde. Hábitos adotados nessa fase da vida poderão acompanhar o indivíduo durante toda a sua vida e, assim, predizer um provável não só seu estilo de vida como também sua condição de saúde na vida adulta. Sabendo que os fatores de risco analisados neste estudo são suscetíveis à modificação, poder identificar de que forma se agregam confere base para intervir.

\section{Conclusão}

Este estudo mostrou que adolescentes brasileiros apresentam agregação de fatores de risco cardiovascular quando são considerados o tabagismo, o consumo de álcool, o sono de curta duração e o excesso de peso. Álcool e tabaco mostraram ser uma combinação importante quando se consideraram as agregações de dois, três ou quatro fatores de risco. Alguns estudos já constatam um aumento da prevalência de fatores de risco cardiovascular em adolescentes. Tal quadro se revela ainda mais preocupante quando se considera a ocorrência de agregação desses fatores, o que implica consequências mais graves para a saúde dessa população. 


\section{Colaboradores}

G. Oliveira elaborou e planejou a estrutura do artigo e o do estudo, a análise de dados, a redação do artigo e a revisão final. T. L. N. Silva colaborou na análise dos dados, redação final e revisão final. I. B. Silva colaborou na redação do artigo. E. S. F. Coutinho colaborou na análise de dados e na revisão final. $\mathrm{K}$. V. Bloch colaborou na revisão final. E. R. A. Oliveira colaborou no planejamento de estudo, estrutura do artigo, redação do artigo e revisão final.

\section{Informações adicionais}

ORCID: Gabriela Oliveira (0000-0002-2403-5094); Thiago Luiz Nogueira da Silva (0000-0003-19611883); Isabel Batista da Silva (0000-0001-57952609); Evandro Silva Freire Coutinho (0000-00024649-7353); Katia Vergetti Bloch (0000-00026992-3159); Elizabete Regina Araujo de Oliveira (0000-0002-6616-4273).

\section{Agradecimentos}

O presente trabalho foi realizado com apoio da Coordenação de Aperfeiçoamento de Pessoal de Nível Superior (Capes; Código de Financiamento 001).

\section{Referências}

1. World Health Organization. Global status report on noncommunicable diseases 2014 http://apps.who.int/iris/bitstream/10665/ 148114/1/9789241564854_eng.pdf (acessado em 30/Set/2017)

2. Santos Filho RD, Martinez TLR. Fatores de risco para doença cardiovascular: velhos e novos fatores de risco, velhos problemas! Arq Bras Endocrinol Metab 2002; 46:212-4.

3. Drager LF, Lorenzi-Filho G, Cintra FD, Pedrosa RP, Bittencourt LR, Poyares D, et al. 1o Posicionamento Brasileiro sobre o Impacto dos Distúrbios de Sono nas Doenças Cardiovasculares da Sociedade Brasileira de Cardiologia. Arq Bras Cardiol 2018; 111:290-341.

4. Burke GM, Genuardi M, Shappell H, D'Agostino Sr. RB, Magnani JW. Temporal associations between smoking and cardiovascular disease, 1971 to 2006 (from the Framingham Heart Study). Am J Cardiol 2017; 120:1787-91.

5. Departamento de Análise de Situação de Saúde, Secretaria de Vigilância Sanitária, Ministério da Saúde. Vigilância de fatores de risco e proteção para doenças crônicas por inquérito telefônico. http://portalarquivos.saude.gov. br/images/pdf/2017/junho/07/vigitel_2016_ jun17.pdf (acessado em 22/Jun/2017).

6. Ohayon M, Wickwire EM, Hirshkowitz M Albert SM, Avidan A, Daly FJ, et al. National Sleep Foundation's sleep quality recommendations: first report. Sleep Health 2017; 3:6-19.

7. Pasch KE, Laska NM, Lytle AL, Moe GS. Adolescent sleep, risk behaviors and depressive symptoms: are they linked? Am J Health Behav 2010; 34:237-48.

8. Mak YW, Wu CS, Hui DW, Lam SP, Tse HY, Yu WY. Association between screen viewing duration and sleep duration, sleep quality, and excessive daytime sleepiness among adolescents in Hong Kong. Int J Environ Res Public Health 2014; 11:11201-19.

9. Matos MG, Gaspar T, Tomé G, Paiva T. Sleep variability and fatigue in adolescents: associations with school-related features. Int J Psychol 2016; 51:323-31.

10. Alamian A, Paradis G. Clustering of chronic disease behavioral risk factors in Canadian children and adolescents. Prev Med 2009; 48:493-9.

11. Cuenca-García M, Huybrechts I, Ruiz JR, Ortega FB, Ottevaere C, González-Gross M, et al. HELENA study group: clustering of multiple lifestyle behaviors and health-related fitness in European adolescents. J Nutr Educ Behav 2013; 45:549-57.

12. Dumith SC, Muniz LC, Tassitano RM, Halla PC, Menezes AMB. Clustering of risk factors for chronic diseases among adolescents from Southern Brazil. Prev Med 2012; 54:393-6.

13. Vasconcellos MTL, Silva PLN, Szklo M, Kuschnir MCC, Klein CH, Abreu GA, et al. Sampling design for the Study of Cardiovascular Risks in Adolescents (ERICA). Cad Saúde Pública 2015; 31:921-30. 
14. Bloch KV, Szklo M, Kuschnir MC, Abreu GA, Barufaldi LA, Klein $\mathrm{CH}$, et al. The Study of Cardiovascular Risk in Adolescents-ERICA: rationale, design and sample characteristics of a national survey examining cardiovascular risk factor profile in Brazilian adolescents. BMC Public Health 2015; 15:94.

15. Silva TLN, Klein CH, Souza AM, Barufaldi LA, Abreu GA, Kuschnir MCC, et al. Participação no Estudo de Riscos Cardiovasculares em Adolescentes - Erica. Rev Saúde Pública 2016; 50 Suppl 1:3s.

16. Figueiredo VC, Szklo AS, Costa LC, Kuschnir MCC, Silva TLN, Bloch KV, et al. ERICA: prevalência de tabagismo em adolescentes brasileiros. Rev Saúde Pública 2016; 50 Suppl 1:12s.

17. Coutinho ESF, França-Santos D, Magliano ES, Bloch, Barufaldi LA, Cunha CF, et al. ERICA: padrões de consumo de bebidas alcoólicas em adolescentes brasileiros. Rev Saúde Pública 2016; 50 Suppl 1:8s.

18. Hirshkowitz M, Whiton K, Albert SM, Alessi C, Bruni O, DonCarlos L, et al. National Sleep Foundation's sleep time duration recommendations: methodology and results summary. Sleep Health 2015; 1:40-3.

19. Bloch KV, Klein CH, Szklo M, Kuschnir MCC, Abreu GA, Barufaldi LA, et al. ERICA: prevalências de hipertensão arterial e obesidade em adolescentes brasileiros. Rev Saúde Pública 2016; 50 Suppl 1:9s.

20. Schuit AJ, van Loon AJ, Tijhuis M, Ocké M. Clustering of lifestyle risk factors in a general adult population. Prev Med 2002; 35:219-24.

21. Instituto Brasileiro de Geografia e Estatística. Pesquisa de Orçamentos Familiares (POF): antropometria e estado nutricional de crianças, adolescentes e adultos no Brasil, 2008-2009. https://biblioteca.ibge.gov.br/visualizacao/li vros/liv45419.pdf (acessado em 17/Ago/2017)

22. Steele CB, Thomas CC, Henley SJ, Massetti GM, Galuska DA, Agurs-Collins T, et al. Vital signs: trends in incidence of cancers associated with overweight and obesity: United States, 2005-2014. MMWR Morb Mortal Wkly Rep 2017; 66:1052-8

23. Zheng Y, Manson JE, Yuan C, Liang MH, Grodstein F, Stampfer MJ, et al. Associations of weight gain from early to middle adulthood with major health outcomes later in life. JAMA 2017; 318:255-69.

24. Mancini MC. Obesidade e doenças associadas. In: Mancini MC, Geloneze B, Salles JEN, Lima JG, Carra MK, organizadores. Tratado de obesidade. Itapevi: AC Farmacêutica; 2010. p. 253-64.
25. Instituto Brasileiro de Geografia e Estatística. Pesquisa Nacional de Saúde do Escolar 2015. https://biblioteca.ibge.gov.br/visualizacao/li vros/liv97870.pdf (acessado em 28/Ago/2017).

26. Taylor B, Rehm J. When risk factors combine: the interaction between alcohol and smoking for aerodigestive cancer, coronary heart disease, and traffic and fire injury. Addict Behav 2006; 31:1522-35.

27. Cureau FV, Duarte P, dos Santos DL, Reichert FF. Clustering of risk factors for noncommunicable diseases in Brazilian adolescents: prevalence and correlates. J Phys Act Health 2014; 11:942-9.

28. Tassitano RM, Dumith SC, Chica DAG, Tenório MCM. Agregamento dos quatro principais comportamentos de risco às doenças não transmissíveis entre adolescentes. Rev Bras Epidemiol 2014; 17:465-78.

29. Oliveira ASD, Moreira NF, Moraes ABV, Pereira RA, Veiga GV. Coocorrência de fatores de risco comportamentais para doenças crônicas não transmissíveis em adolescentes: prevalência e fatores associados. Rev Nutr 2017; 30:747-58.

30. Poortinga W. The prevalence and clustering of four major lifestyle risk factors in an English adult population. Prev Med 2007; 44:124-8.

31. Seo YG, Choi MK, Kang JH, Lee HJ, Jang HB, Park SI, et al. Cardiovascular disease risk factor clustering in children and adolescents: a prospective cohort study. Arch Dis Child 2018; 103:968-73.

32. Brito ALS, Hardman CM, Barros MVG. Prevalência e fatores associados à simultaneidade de comportamentos de risco à saúde em adolescentes. Rev Paul Pediatr 2015; 33:423-30.

33. Silva KS, Lopes SA, Vasques DG, Costa FF, Silva RCR. Clustering of risk factors for chronic noncommunicable diseases among adolescents: prevalence and associated factors. Rev Paul Pediatr 2012; 30:338-45.

34. Plotnikoff RC, Karunamuni N, Spence JC, Storey $\mathrm{K}$, Forbes $\mathrm{L}$, Raine $\mathrm{K}$, et al. Chronic disease-related lifestyle risk factors in a sample of Canadian adolescents. J Adolesc Health 2009; 44:606-9.

35. Farias Júnior JC, Nahas MV, Barros MVG, Loch MR, Oliveira ESA, De Bem MFL, et al. Comportamentos de risco à saúde em adolescentes no Sul do Brasil: prevalência e fatores associados. Rev Panam Salud Pública 2009; 25:344-52.

36. Wu J, Wu H, Wang J, Guo L, Deng X, Lu C. Associations between sleep duration and overweight/obesity: results from 66.817 Chinese adolescents. Sci Rep 2015; 5:16686. 


\section{Abstract}

This study aimed to analyze the aggregation of alcohol consumption, smoking, excess weight, and short sleep in Brazilian adolescents. This was a cross-sectional multicenter study conducted with teens participating in the Study of Cardiovascular Risk Factors in Adolescents (ERICA in Portuguese). The sample consisted of adolescents that answered the complete questionnaires on sleep, tobacco, and alcoholic beverages, in addition to having their weight and height measured. Aggregation was analyzed by comparing the observed and expected prevalence of risk factors in all possible groupings, with the respective $95 \%$ confidence intervals. Analyses were performed in Stata 14 using the svy (survey) command for complex sample data. The sample included 73,624 adolescents, of whom $25.5 \%$ had excess weight and $24.2 \%$ consumed alcoholic beverages. Aggregation of the four risk factors was $O / E=5$.6. Aggregation of three factors was more prevalent in those 15 to 17 years of age $(P=4.8)$. In the POR (prevalence odds ratio) analysis of the combination of two risk factors, those that smoked showed 11.80 higher odds of also consuming alcohol, compared to those that did not smoke, and vice versa, in private schools. In relation to age, adolescents 12 to 14 years of age that smoked showed 15.46 times higher odds of also drinking, and vice and versa. Adolescents in the sample presented the aggregate presence of four risk factors, and there was a significant relationship between tobacco and alcohol consumption.

Adolescent; Alcoholic Beverages; Tobacco; Sleep; Risk Factors

\section{Resumen}

El objetivo fue analizar la agregación del consumo de alcohol, tabaco, exceso de peso y sueño corto en adolescentes brasileños. Se trata de un estudio transversal, multicéntrico, realizado a partir de adolescentes participantes del Estudio de Riesgos Cardiovasculares en Adolescentes (ERICA). La muestra estuvo compuesta por adolescentes que respondieron completamente los cuestionarios sobre sueño, tabaco y uso de bebidas alcohólicas, además de haber realizado antropometría con medidas de peso y estatura medidas. La agregación se analizó comparando la prevalencia observada con la esperada de los factores de riesgo en todas las posibilidades de agrupamiento, y sus intervalos de confianza (95\%). Los análisis se realizaron con el programa estadístico Stata 14, mediante el uso del control svy (survey) para datos de muestra compleja. Son 73.624 adolescentes, un 25,5\% de los estudiantes presentan exceso de peso y un 24,2\% consumen bebidas alcohólicas. La agregación de los cuatro factores de riesgo fue de $\mathrm{O} / \mathrm{E}=5,6 \mathrm{La}$ prevalencia de agregación de los tres factores fue mayor en aquellos con 15 a 17 años $(P=4,8)$. En el análisis de ORP (odds ratio de prevalencia) de la combinación de dos factores de riesgo, se observó que aquellos que fuman tienen 11,80 veces más oportunidades de beber también, cuando los comparamos con aquellos que no fuman y viceversa en las escuelas privadas. En relación con la edad, los adolescentes con 12 y 14 años que fuman tienen 15,46 veces más oportunidades de también beber y viceversa. Los adolescentes estudiados presentan la presencia de los cuatro factores de forma agregada y existe una relación significativa entre tabaco y el consumo de alcohol.

Adolescente; Bebidas Alcohólicas; Tabaco; Sueño; Factores de Riesgo
Recebido em 21/Nov/2018

Versão final reapresentada em 28/Mai/2019

Aprovado em 17/Jun/2019 